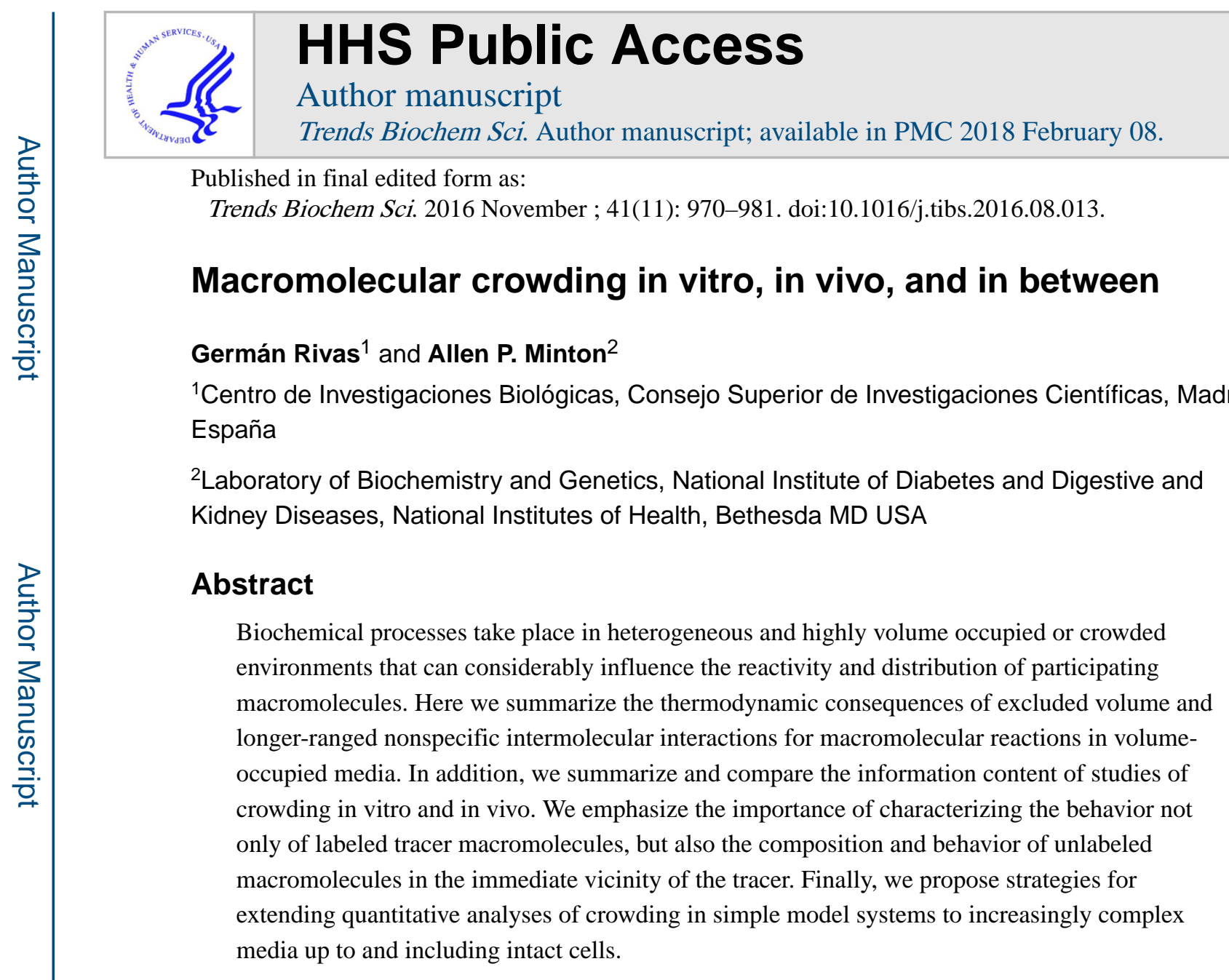

\title{
Keywords
}

steric interactions; long range interactions; nonspecific interactions; crowding theory; in-cell experiments

\section{What is macromolecular crowding?}

The term "macromolecular crowding" was originally used to connote the influence of a high total weight per volume concentration of macromolecules on the equilibria and kinetics of macromolecular reactions taking place in a highly volume-occupied or "crowded" solution, independent of whether any single species of reactant or product was concentrated ${ }^{1}$. The observation that almost every biological fluid medium, both extracellular and intracellular, contains a substantial total concentration of macromolecules has led to a widespread hope and expectation that an understanding of the physical-chemical origins of crowding effects will help us to understand how biochemical reactions within a living organism may differ, and by how much, from those observed in a test tube $\mathrm{e}^{2-5}$. It is however necessary to recognize that all differences between specified biochemical equilibria and rates within an intact cell and the same equilibria and rates in a dilute solution of purified macromolecules cannot and should not be attributed to crowding. One cannot ignore the likelihood of additional modulating effects deriving from interactions of soluble macromolecules with membranes and other structural elements ${ }^{6-9}$, in addition to unidentified or incompletely characterized interactions between the studied reactants and other soluble species such as 
molecular chaperones ${ }^{10}$. Thus we would like to make clear at the outset that the term macromolecular crowding should be used to describe only those effects arising from the presence of a total high weight per volume concentration of functionally unrelated soluble macromolecules.

The influence of crowding on macromolecular equilibria has traditionally been studied through the use of simple theoretical and experimental models that may be explored in quantitative detail. The motivation for this approach has been the idea that only when simple systems are well understood, should one attempt to add and study theoretical and/or experimental elements of additional complexity in a stepwise fashion, with the goal of eventually reaching an understanding of systems that mimic physiological media. This approach has been described as "bottom-up"11. In contrast, a growing number of recent publications describe attempts to characterize the effects of crowding on macromolecular equilibria and kinetics directly within intact cells. One might describe such an approach as "top-down", although the term must be qualified, as discussed subsequently. The purpose of this critical review is to compare the information content of the two approaches and to suggest how future progress may be optimized by a combination of both.

\section{What have we learned from theory and in vitro experiment?}

The basic thermodynamic relations governing the effects of crowding on macromolecular equilibria are summarized in Box 1. The effects of crowding on equilibria, kinetics and transport properties have been more completely described elsewhere ${ }^{12-16}$. It follows from the relations presented in Box 1 that in order to understand the physical basis of an observed crowding effect, information about the free energies of transfer of reactants and products from a dilute to a crowded medium is essential.

The free energy of transfer is essentially equal to the free energy of interaction between a molecule of the newly introduced species (termed probe) and all of the other macromolecules (termed crowders) at equilibrium in the crowded medium ${ }^{17}$. The free energy of transfer depends upon the effective potential of interaction between the probe and crowder molecules in solution, the nature of which may be deduced from the composition dependence of various solution properties (see for example ${ }^{18-21}$ ).

From the results of many in vitro experiments conducted upon purified macromolecules under precisely specified conditions we have learned that the total free energy of interaction between a particular probe or trace species at low concentration and a particular crowding species at arbitrarily high concentration is the sum of several contributions. The most basic and often dominant contribution, called volume exclusion, is an entropic effect arising from the mutual impenetrability of macromolecules (Figure 1). The effects of excluded volume upon transfer free energies become evident only when the fraction of total volume occupied by macromolecules becomes substantial and when the size of the probe species is comparable to or greater than the size of the concentrated or crowder species ${ }^{13}$. The contribution of other longer-ranged interactions to free energies of transfer and chemical equilibria ${ }^{12}$ will be discussed subsequently. 
Early theoretical treatments of the effect of "crowding" or high total macromolecular volume occupancy upon macromolecular reactions focused upon universal contribution of volume exclusion arising from mutual impenetrability of reactants, products and crowding species to the free energies of transfer ${ }^{22-24}$. This is because steric repulsion is undeniably the most universal form of nonspecific interaction ${ }^{25}$. Simple models for estimating the free energies of transfer of macromolecules from a dilute to a crowded solution were based upon the recognition that the effective potential acting between compact globular proteins in solution could be approximated by a hard particle potential acting between equivalent convex particles (i.e., no interaction beyond contact distances) ${ }^{12}$. Well-developed theories of convex hard particle fluids ${ }^{26-28}$ were then employed to estimate the free energy associated with the introduction of a tracer species into a fluid consisting of one or more crowder species at arbitrary concentration. Oosawa and Asakura had earlier theoretically predicted that a high concentration of macromolecules would induce an effective attraction between larger colloidal particles ${ }^{29,30}$, and their results are equivalent to those of excluded volume theory in the limit of low crowder concentration. However, the formalism of Oosawa and Asakura does not provide a convenient quantitative description of the effect of excluded volume on chemical reactions and so far has not been utilized for that purpose.

Experimental studies carried out on many model systems designed to reduce the effect of nonspecific interactions other than volume exclusion have shown that the hard particle model provides a powerful qualitative, semi-quantitative and often quantitative means for prediction and analysis of crowding effects in these systems. Earlier studies have been reviewed in ${ }^{13-15}$, and recent studies include ${ }^{31-35}$. At the same time, experimental measurement of the $\mathrm{pH}$ dependence of colligative properties of protein solutions revealed that under other conditions, longer-ranged repulsive and attractive electrostatic interactions between macromolecules contribute significantly to the chemical potential, and hence reactivity, of macromolecules in concentrated solutions ${ }^{36,37}$. Nonspecific electrostatic attraction between probe and crowder species was found to counteract the effects of volume exclusion to a varying extent, and in certain instances to dominate the overall behavior of individual tracer proteins, peptides, and nucleotides ${ }^{19,38-40}$. Several approaches to the generalization of crowding theory to allow for enthalpic as well as entropic intermolecular interactions have been published $21,36,38,41-46$. While these generalized theories can be used to rationalize experimental results, they are not yet capable of quantitatively predicting the effect of a specific macromolecular crowding species upon a specific macromolecular reaction.

Finally, preliminary in vitro studies indicate that the effects of mixed crowders (protein + polymer, polymer + DNA, protein + protein,) on the stability and aggregation propensity of tracer proteins may be far from additive of the effects of each individual crowder ${ }^{47-49}$. Thus the study of crowding has become more complex and experimentally challenging as we recognize that the nonspecific intermolecular interactions underlying crowding effects depend not only upon the abundances and gross sizes and shapes of probe and crowder species, but also upon their chemical compositions and environmental variables. 
In Box 2 we list a number of conditions that should be satisfied if the results of any crowding experiment conducted in vitro are to be amenable to quantitative analysis. We believe that this should serve as a checklist when such experiments are designed.

\section{What have we learned from "in vivo" experiments?}

Among life scientists, the chief motivation for studying the properties of crowded media is to understand how crowding may impact macromolecular reactions in physiological media. Estimates of the total concentration of macromolecules (proteins, nucleic acids, polysaccharides) in a cell range from 200 to over $400 \mathrm{~g} / \mathrm{l}^{50-52}$, but these estimates are extremely rough and do not distinguish between mobile or diffusible species and immobile species incorporated into static structural elements. The general strategy employed by investigators studying "in cell crowding" is to introduce fluorescently or isotopically labeled tracer proteins into the cell by means of transfection, microinjection, or recombinant expression. The kinetics and/or equilibria of reactions involving the tracer species are then characterized by fluorescence microscopy or NMR spectroscopy ${ }^{53-59}$.

Although the intact cell presents an attractive system for the study of "crowding in vivo", the interpretation of results obtained from such studies must take into account complexity and heterogeneity of the cellular interior. Even the simplest of prokaryotic organisms provides a variety of microenvironments that are sufficiently distinct in composition (Figure 2) so that a single type of probe molecule within each of these microenvironments may exhibit significantly different transport and thermodynamic properties, which may derive not only from bulk crowding, but confinement ${ }^{60}$, adsorption ${ }^{6}$ and liquid-liquid phase separation ${ }^{61,62}$ as well. The interior of a eukaryotic cell is far more complex, containing a variety of membrane-enclosed organelles, as well as a network of cytoskeletal fibers. The relative contributions of crowding, confinement, and adsorption to macromolecular reactivity will likely differ between each of the intracellular microenvironments.

In the original "in cell" experiments aimed at characterizing the stability of a particular protein, the labeled probe molecule was introduced into the cell, but no information was available regarding the distribution of the probe among microenvironments that may differ widely in composition. The signal obtained from the labeled probe (or probes) was thus treated as a whole cell average. Such treatment is problematic for at least two reasons. (1) If the probe is limited to a single microenvironment, the signal observed reflects only the behavior of the probe in that unidentified microenvironment. (2) If the probe is distributed between multiple microenvironments, the cell average signal may not reflect the behavior of the probe in any of the individual microenvironments, or may reflect the behavior of the probe primarily in the microcompartments into which it preferentially distributes.

Technical advances in fluorescence microscopy have provided the ability to measure a probe signal as a function of location within an intact cell ${ }^{55,56,63}$. Experiments conducted using this technique have shown clearly that the equilibrium thermal stability and kinetics of conformational change of a given fluorescently labeled probe can vary substantially within a single cell ${ }^{64,65}$, and between cells of the same type ${ }^{39}$. For example, the positional variability of folding kinetics and equilibrium of a mutant of phosphoglycerokinase fused to green and 
red fluorescent proteins in intact U2OS (mammalian) cells is clearly non-random (Figures $3 \mathrm{~A}, \mathrm{~B})$, and appears to correlate with supramolecular structures such as membranes or cytoskeletal fibers within the cell ${ }^{55}$. This could indicate that the in-cell behavior of a probe molecule may be influenced by its interaction with surfaces as much as by crowding in the bulk medium, as has been suggested previously $6,8,66,67$.

Although fluorescence microscopy has provided enhanced spatial resolution, the origin of the observed heterogeneous behavior remains indeterminate, since the positional and temporal variations in the local composition of unlabeled macromolecules and their states of association remain largely unknown. In addition, when measuring thermal stability of a fluorescent probe within the cell, one cannot neglect the possibility of changes in the association state and abundance of unlabeled crowding macromolecules that may be either incidental to or associated with a homeostatic response of the living cell to changes in temperature. Finally, recent studies have shown that the results of in-cell experiments are subject to qualitative variation with the choice of fluorescent label ${ }^{68}$ and choice of probe molecule 69,70 .

Further complexity becomes evident when one considers the possibility that total macromolecular concentration, a strong modulator of the magnitude of the crowding effect, may vary with location within the cell, since osmolarity may be maintained by compensatory variation in electrolyte concentration. Even within regions of roughly constant total macromolecular concentration, a local microenvironment is likely to contain multiple crowding species, some of which may have a net repulsive interaction with the probe, and some of which may have a net attractive interaction with the probe. It follows that if the probe is free to distribute itself within the cellular interior, then at equilibrium it will tend to accumulate preferentially in the most attractive (oppositely charged) and/or the least crowded microenvironments. If this is the case, the probe will not report crowding effects in the most volume-excluded and/or like-charged microenvironments, where the consequences of crowding are likely to be greatest.

Measurements of fluorescence resonance energy transfer (FRET) and fluorescence correlation and cross-correlation have also been used, to characterize the kinetics and equilibria of binary heteroassociation of pairs of proteins bearing donor and acceptor labels ${ }^{58,59}$. In addition, FRET-based "crowding sensors" consisting of donor-acceptor pairs linked by a flexible polymer ${ }^{56}$ or a polypeptide consisting of two helical regions linked by a flexible "hinge" 54 have been designed. It is expected that the intensity of observed FRET, which is a function of the average distance between the donor and acceptor, will be a measure of the degree to which a particular crowded environment promotes or inhibits association of the donor/acceptor pair (contraction of the polymer chain or closing of the "hinge").

Many of the complications and uncertainties associated with the interpretation of in cell measurements of thermal stability discussed earlier in this section apply to the interpretation of the results of these studies of association as well. In one study of hetero-association of probe molecules in intact cells, the authors reported "significant differences in protein mobility and $\mathrm{K}_{\mathrm{d}}$ [dissociation equilibrium constant]" for the identical association reaction 
examined in two different cell types. The latter result is not at all surprising since it is well established that variations in the size, abundance and chemical identity of crowding species have a major influence upon the magnitude of the crowding effect on protein association $^{43,48,49}$. Thus conclusions drawn from a study of the behavior of a single set of probe reactants in a single cell, even if correct for the particular system studied, cannot be assumed to apply to a different reaction in a different microenvironment within the same cell, another cell of the same type, or in another cell type.

\section{How can we connect bottom-up (in vitro) and top-down (in vivo) observations?}

The above considerations do not invalidate the potential value of in-cell measurements. However, one must keep in mind that such experiments in and of themselves do not constitute a "top-down" analysis, since they are only the first step in such an analysis. Future progress depends upon moving from the top down and from the bottom up.

First, methods should be developed that will permit fractionation of cell contents in a way that preserves as well as possible each of the various microenvironments. Such fractionation is probably more readily accomplished with eukaryotic cells containing discrete organelles ${ }^{71}$, but even in E. coli the nucleoid can be separated from the proteinaceous cytoplasm, and the remaining soluble proteins and macromolecular complexes separated from membranous environments ${ }^{72}$.

Second, chemical and/or proteomic analysis of the contents of a nominally homogeneous microcompartment should be employed to provide information about the identity and abundance of major macromolecular crowder species, and biophysical techniques ${ }^{73,74}$ should be employed to ascertain their states of association in situ.

Third, control experiments should be conducted to ensure that probe labels do not introduce artifactual interactions between probe and the environment. The behavior of different probe species and differences in probe labeling, including exchange of the position of donor and acceptor fluorophores used in a FRET measurement $68,75,76$, should be characterized within a single uniform microenvironment in order to determine whether probe-independent conclusions may be drawn from the experimental results.

Fourth, the equilibrium behavior of the selected probe or probes should be characterized within individual isolated microcompartments. We do not believe that whole cell lysate is a satisfactory substitute for fractionated organelles, since the process of lysis artificially homogenizes cell contents, mixing macromolecules that are not present together in intact cells.

Fifth, the behavior of the selected probe or probes should then be characterized in the presence of individual macromolecular constituents of the microcompartment at their "invivo" abundances and well-defined mixtures of these constituents. These studies should include the influence on probe behavior of probe-accessible membranes and cytoskeletal fibers if present ${ }^{8,77}$. Attempts should be made to determine the minimal composition of the 
partially reconstituted "semi-synthetic" microenvironment in which the behavior of the probe is comparable or identical to that observed in the intact microenvironment?

Sixth, attention should be paid to the presence and abundance of molecules like small heat shock proteins within a particular environment, that preferentially bind to partially unfolded proteins ${ }^{10,78}$ and could thus promote overall destabilization of a probe.

And finally, in a medium as complex as the interior of an intact cell it is absolutely necessary to distinguish modulation of biochemical reactions arising from macromolecular crowding from other modulating effects such as the nonspecific interaction of proteins with membranes or cytoskeletal structures ${ }^{8,79}$, and the partitioning of reactants between immiscible phases ${ }^{80}$.

\section{Concluding Remarks and Future Perspectives}

To date, most measurements of probe protein behavior within cells have been aimed at characterizing the stability and dynamics of the probe molecule. The results of such experiments are in many cases not susceptible to quantitative interpretation due to our lack of knowledge of the structure of the unfolded state (or manifold of unfolded states) of the probe protein, and how structure and transfer free energy of the unfolded state are affected by both excluded volume and attractive interactions with the crowded environment. Moreover, the global unfolding and refolding of proteins within cells is likely controlled by strong interactions with molecular chaperones and accessory proteins rather than by thermodynamic equilibrium ${ }^{10,81}$, so the biological relevance of studies of thermodynamic stability within cells is unclear.

We submit that experiments aimed at measuring the effects of crowding on reversible associations of native probes are not only more relevant from a biological standpoint than measurements of protein stability, but also simpler to interpret, due to the serious theoretical difficulties associated with quantitative analysis of interactions between flexible unfolded macromolecules and rigid macromolecules. Furthermore, properly designed probe-based measurements of self- and/or heteroassociation can be more sensitive to crowding effects than measurements of stability. For example, several measurements, simulations, and theoretical estimates ${ }^{82-85}$ have reported only small effects of crowding on the formation of homo- or hetero-dimers in solutions of comparably sized crowding molecules (i.e., increases in equilibrium association constants by factors of between 2 and 5 , or $-0.5<\Delta \Delta G / R T<$ $-1.5)$. However, binary hetero-association results in the formation of a single protein-protein contact interface, whereas the self-assembly of a larger oligomer (e.g., $n P \rightleftarrows P_{n}$ where $n>$ 2) results in the formation of a minimum of $n-1$ intersubunit interfaces and possibly more, as in the case of a tetragonal tetramer with 6 intersubunit interfaces. When one multiplies the individually small crowding bias toward the formation of a single interface by the number of interfaces, the enhancement of oligomer formation by crowding can become very large. It follows that an experiment designed to follow the concerted formation of a large oligomer would be expected to be much more sensitive to excluded volume effects than one designed to follow the formation of a homo- or heterodimer. Large changes in self-association equilibria were indeed observed in studies of the effect of crowding on the concerted 
formation of hexamers of $\mathrm{ClpB}^{34}$, heptamers of $\mathrm{cp} 10^{32}$ and decamers of bovine pancreatic trypsin inhibitor ${ }^{86}$.

Several of the reported studies of probe stability within crowded media utilized probes that are smaller than the predominant crowding species ${ }^{57,87-89}$. Such experiments should not be expected to be sensitive to excluded volume effects. Excluded volume theory predicts that the contribution of excluded volume to a particular reaction equilibrium will only become substantial when the size of the probe is comparable to or exceeds that of the major crowding species ${ }^{13}$ and when the probe reaction (isomerization or association) is accompanied by a substantial reduction of the ratio of solvent-exposed surface to volume ${ }^{12}$. These predictions are borne out by the following observations: (1) The formation of polymers of sickle hemoglobin ( $\mathrm{HbS}$ ) is facilitated by high concentrations of bovine serum albumin and hemoglobin mutants that do not coprecipitate with $\mathrm{HbS}^{90}$; (2) the formation of amyloid fibers of alpha synuclein is strongly promoted by high concentrations of other proteins as well as by nonionic polymers ${ }^{91}$; and (3) the formation of fiber bundles of FtsZ is strongly facilitated not only by the addition of traditional polymeric crowding agents, but also by the addition of $10 \%$ (by volume) of BSA, ovomucoid, and Escherichia coli lysate ${ }^{92}$.

In view of the possible variation of the factors known to contribute to tracer - crowder interactions, it is evident that information obtained from measurements on a specific probe in a single (micro)environment under a fixed set of experimental conditions cannot be reliably generalized. When the abundances and chemical compositions of the interacting molecules and experimental conditions are significantly different from those studied, such generalization would likely be erroneous and misleading in the absence of independently obtained evidence to the contrary.

Although recognized long ago $66,79,93,94$, the importance of weak, nonspecific interactions as modulators of biochemical processes in vivo has only recently begun to be widely appreciated ${ }^{5}, 11,65,95$. Although emphasis has been placed upon attractive interactions and the formation of weakly associated complexes, it is important to keep in mind that some of the most influential weak, nonspecific interactions that have been identified as significant modulators of biochemical equilibria and rates are repulsive steric and electrostatic interactions between macromolecules that do not form complexes.

We believe that future advances in our understanding of the influence of macromolecular crowding upon rates and equilibria of macromolecular reactions in media as complex and heterogeneous as the interior of a living cell will depend less upon the development of new tools than upon the willingness of researchers to utilize experimental and theoretical tools already in hand to build up from the bottom and, perhaps even more importantly, to build down from the top.

\section{Acknowledgments}

We thank Damien Hall (Australian National University) and Martin Gruebele (Univ. of Illinois) for useful discussions, and Dr. Gruebele for Figure 3B. Research of GR is supported by the Spanish Government through grant BFU2014-52070-C2-2-P and that of APM is supported by the Intramural Research Program of the National Institute of Diabetes and Digestive and Kidney Diseases. 


\section{REFERENCES}

1. Minton AP, Wilf J. Effect of macromolecular crowding upon the structure and function of an enzyme: glyceraldehyde-3-phosphate dehydrogenase. Biochemistry. 1981; 20:4821-4826. [PubMed: 7295652]

2. Record MT Jr, et al. Biophysical compensation mechanisms buffering E. coli protein-nucleic acid interactions against changing environments. Trends in Biochemical Sciences. 1998; 23:190-194. [PubMed: 9612084]

3. Minton AP. How can biochemical reactions within cells differ from those in test tubes? J. Cell Sci. 2006; 119:2863-2869. [PubMed: 16825427]

4. Theillet F-X, et al. Physicochemical properties of cells and their effects on intrinsically disorded proteins. Chem. Revs. 2014; 114:6661-6714. [PubMed: 24901537]

5. Chien P, Gierasch LM. Challenges and dreams: physics of weak interactions essential to life. Molecular Biology of the Cell. 2014; 25:3474-3477. [PubMed: 25368424]

6. Minton AP. Confinement as a determinant of macromolecular structure and reactivity. II. Effects of weakly attractive interactions between confined macrosolutes and confining structures. Biophys. J. 1995; 68:1311-1322. [PubMed: 7787020]

7. Herrig A, et al. Cooperative adsorption of ezrin on PIP2-containing membranes. Biochemistry. 2006; 45:13025-13034. [PubMed: 17059219]

8. Knull H, Minton AP. Structure within eukaryotic cytoplasm and its relationship to glycolytic metabolism. Cell Biochem. Func. 1996; 14:237-248.

9. Langdon BB, et al. Interfacial protein-protein associations. Biomacromolecules. 2014; 15:66-74. [PubMed: 24274729]

10. Clerico EM, et al. How hsp70 molecular machines interact with their substrates to mediate diverse physiological functions. J. Mol. Biol. 2015; 427:1575-1588. [PubMed: 25683596]

11. Gierasch LM, Gershenson A. Post-reductionist protein science, or putting Humpty Dumpty back together again. Nature Chemical Biology. 2009; 5:775-777.

12. Minton AP. The effect of volume occupancy upon the thermodynamic activity of proteins: some biochemical consequences. Molecular and Cellular Biochemistry. 1983; 55:119-140. [PubMed: 6633513]

13. Hall D, Minton AP. Macromolecular crowding: qualitative and semiquantitative successes, quantitative challenges. Biochim Biophys Acta. 2003; 1649:127-139. [PubMed: 12878031]

14. Zhou H-X, et al. Macromolecular crowding and confinement: biochemical, biophysical, and potential physiological consequences. Ann. Rev. Biophys. 2008; 37:375-397. [PubMed: 18573087]

15. Zimmerman SB, Minton AP. Macromolecular crowding: biochemical, biophysical, and physiological consequences. Ann. Rev. Biophys. Biomol. Struct. 1993; 22:27-65. [PubMed: 7688609]

16. Minton, AP. Macromolecular crowding: effects on association equilibria and kinetics. In: Roberts, GCK., editor. Encyclopedia of Biophysics. Springer-Verlag; 2013. p. 1338-1342.

17. Widom B. Some topics in the theory of fluids. J. Chem. Phys. 1963; 39:2808-2812.

18. Fernández C, Minton AP. Effect of non-additive repulsive interactions upon the light scattering of concentrated protein-osmolyte mixtures. J. Phys. Chem. B. 2011; 115:1289-1293. [PubMed: 21175126]

19. Fodeke AA, Minton AP. Quantitative characterization of polymer-polymer, protein-protein, and polymer-protein interaction via tracer sedimentation equilibrium. J. Phys. Chem. B. 2010; 114:10876-10880. [PubMed: 20677765]

20. Minton AP. Effective hard particle model for the osmotic pressure of highly concentrated binary protein solutions. Biophys. J. 2008; 93:L57-L59.

21. Knowles DB, et al. Chemical interactions of polyethylene glycols (PEGs) and glycerol with protein functional groups: applications to effects of PEG and glycerol on proein processes. Biochemistry. 2015; 54:3528-3542. [PubMed: 25962980] 
22. Laurent TC, et al. Conformational transitions of polynucleotides in polymer media. Eur. J. Biochem. 1974; 43:231-235. [PubMed: 4275655]

23. Minton AP. Excluded volume as a determinant of macromolecular structure and reactivity. Biopolymers. 1981; 20:2093-2120.

24. Nichol LW, et al. Effect of inert polymers on protein self-association. FEBS Lett. 1981; 126:18-20. [PubMed: 7238860]

25. Ellis RJ. Macromolecular crowding: obvious but underappreciated. Trends Biochem. Sci. 2001; 26:597-604. [PubMed: 11590012]

26. Boublík T. Statistical thermodynamics of convex molecule fluids. Mol. Phys. 1974; 27:1415-1427.

27. Cotter MA. Hard spherocylinders in an anisotropic mean field: a simple model for a nematic liquid crystal. J. Chem. Phys. 1977; 66:1098-1106.

28. Lebowitz JL, et al. Scaled particle theory of fluid mixtures. J. Chem. Phys. 1965; 43:774-779.

29. Asakura S, Oosawa F. On interaction between two bodies immersed in a solution of macromolecules. J. Chem. Phys. 1954; 22:1255-1256.

30. Asakura S, Oosawa F. Interaction between particles suspended in solutions of macromolecules. J. Polym. Sci. 1958; 33:183-192.

31. Aden J, Wittung-Stafshede P. Folding of an unfolded protein by macromolecular crowding in vitro. Biochemistry. 2014; 53:2271-2277. [PubMed: 24665900]

32. Aguilar X, et al. Macromolecular crowding extended to a heptameric system: the co-chaperonin protein 10. Biochemistry. 2011; 50:3034-3044. [PubMed: 21375247]

33. Christiansen A, Wittung-Stafshede P. Synthetic crowding agent dextran causes excluded volume interactions exclusively to tracer protein azurin. FEBS Lett. 2014; 588:811-814. [PubMed: 24491997]

34. Alfonso C, et al. Sedimentation equilibrium analysis of clpB self-association in diluted and crowded solutions. Meth. Enzymol. 2015; 562:135-160. [PubMed: 26412650]

35. Stiehl O, et al. Macromolecular crowding impacts on the diffusion and conformation of DNA hairpins. Phys. Rev. E. 2015; 91:012703.

36. Minton AP. A molecular model for the dependence of the osmotic pressure of bovine serum albumin upon concentration and pH. Biophys. Chem. 1995; 57:65-70. [PubMed: 8534837]

37. Minton AP, Edelhoch H. Light scattering of bovine serum albumin solutions: extension of the hard particle model to allow for electrostatic repulsion. Biopolymers. 1982; 21:451-458.

38. Jiao M, et al. Attractive protein-polymer interactions markedly alter the effect of macromolecular crowding on protein association equilibria. Biophys. J. 2010; 99:914-923. [PubMed: 20682270]

39. Gao M, et al. RNA hairpin folding in the crowded cell. Angewandte Chemie Intl. Ed. 2016; 55:3224-3228.

40. Sarkar M, et al. Protein crowder charge and protein stability. Biochemistry. 2014; 53:1601-1606. [PubMed: 24552162]

41. Schellman JA. Protein stability in mixed solvents: a balance of contact interaction and excluded volume. Biophys. J. 2003; 85:108-125. [PubMed: 12829469]

42. Douglas JF, et al. Crowding induced self-assembly and enthalph-entropy compensation. Phys. Rev. Lett. 2009; 103:135701. [PubMed: 19905522]

43. Minton AP. Quantitative assessment of the relative contributions of steric repulsion and chemical interactions to macromolecular crowding. Biopolymers. 2013; 99:239-244. [PubMed: 23348671]

44. Sapir L, Harries D. Origin of enthalpic depletion forces. J. Phys. Chem. Lett. 2014; 5:1061-1065. [PubMed: 26274449]

45. Sapir L, Harries D. Macromolecular stabilization by excluded cosolutes: mean field theory of crowded solutions. J. Chem. Theory Comput. 2015; 11:3478-3490. [PubMed: 26575781]

46. Shkel IA, et al. Separating chemical and excluded volume interactions of polyethylene glycols with native proteins: comparison with PEG effects on DNA helix formation. Biopolymers. 2015; 103:517-527. [PubMed: 25924886]

47. Du F, et al. Mixed macromolecular crowding accelerates the refolding of rabbit muscle creatine kinase: implications for protein folding in physiological environments. J. Mol. Biol. 2006; 364:469-482. [PubMed: 17027032] 
48. Zhou B-R, et al. Mixed macromolecular crowding inhibits amyloid formation of hen egg lysozyme. Biochim. Biophys. Acta. 2009; 1784:472-480.

49. Monterroso B, et al. Charged molecules modulate the volume exclusion effects exerted by crowders on FtsZ polymerization. PLoS ONE. 2016; 11:e0149060. [PubMed: 26870947]

50. Fulton AB. How crowded is the cytoplasm? Cell Biochem. Func. 1982; 30:345-347.

51. McGuffee SR, Elcock AH. Diffusion, crowding \& protein stablity in a dynamic molecular model of the bacterial cytoplasm. PLoS Comput. Biol. 2010; 6:e1000694. [PubMed: 20221255]

52. Zimmerman SB, Trach SO. Estimation of macromolecule concentrations and excluded volume effects for the cytoplasm of Escherichia col. J. Mol. Biol. 1991; 222:599-620. [PubMed: 1748995]

53. Ignatova $\mathrm{Z}$, et al. From the test tube to the cell: exploring the folding and aggregation of a betaclam protein. Peptide Science. 2007; 88:157-163. [PubMed: 17206628]

54. Boersma AJ, et al. A sensor for quantification of macromolecular crowding in living cells. Nature Methods. 2015; 12:227-229. [PubMed: 25643150]

55. Ebbinghaus $\mathrm{S}$, et al. Protein folding stability and dynamics imaged in a living cell. Nature Methods. 2010; 7:319-323. [PubMed: 20190760]

56. Gnutt D, et al. Excluded volume effects in living cells. Angew Chem Int. 2015; 54:2548-2551.

57. Smith AE, et al. In-cell thermodynamics and a new role for protein surfaces. Proc Natl Acad Sci USA. 2016; 113:1725-1730. [PubMed: 26755596]

58. Sudhaharan T, et al. Determination of in vivo dissociationa constant Kd of Cdc42-effector complexes in live mammalian cells using single wavelength cross-correlation spectroscopy. J. Biol. Chem. 2009; 284:13602-13609. [PubMed: 19293156]

59. Phillip Y, et al. Protein binding dynamics imaged in a living cell. Proc Natl Acad Sci USA. 2012; 109:1461-1466. [PubMed: 22307600]

60. Minton AP. Confinement as a determinant of macromolecular structure and reactivity. Biophys. J. 1992; 63:1090-1100. [PubMed: 1420928]

61. Walter H, Brooks DE. Phase separation in cytoplasm, due to macromolecular crowding, is the basis for microcompartmentation. FEBS Lett. 1995; 361:135-139. [PubMed: 7698310]

62. Keating CD. Aqueous phase separation as a possible route to compartmentalization of biological molecules. Acc. Chem. Res. 2012; 18:2114-2124.

63. Guzman I, Gruebele M. Protein folding dynamics in the cell. J Phys Chem B. 2014; 118:84598470. [PubMed: 24878167]

64. Ebbinghaus S, Gruebele M. Protein folding landscapes in the living cell. J Phys Chem Lett. 2011; 2:314-319.

65. Wirth AJ, Gruebele M. Quinary protein structure and the consequences of crowding in living cells: leaving the test-tube behind. Bioessays. 2013; 35:904-993.

66. Minton, AP. Holobiochemistry: an integrated approach to the understanding of biochemical mechanism that emerges from the study of proteins and protein associations in volume-occupied solution. In: Srere, PA., et al., editors. Structural and Organizational Aspects of Metabolic Regulation. Wiley-Liss; 1990. p. 291-306.

67. Hoppe T, Minton AP. An equilibrium model for the combined effect of macromolecular crowding and surface adsorption on the formation of linear protein fibrils. Biophys. J. 2015; 108:957-966. [PubMed: 25692600]

68. Dave K, et al. The effect of fluorescent protein tags on phosphoglycerate kinase stability is nonadditive. J Phys Chem B. 2016; 120:1061-1068.

69. Dhar A, et al. Protein stability and folding kinetics in the nucleus and endoplasmic reticulum of eucaryotic cells. Biophys. J. 2011; 101:421-430. [PubMed: 21767495]

70. Guzman I, et al. The extracellular protein VLsE is destabilized inside cells. J Mol Biol. 2014; 426:11-20. [PubMed: 24013077]

71. Kempner ES, Miller JH. The molecular biology of Euglena Gracilias IV. Cellular stratification by centrifuging. Exp. Cell Res. 1968; 51:141-149. [PubMed: 4173660]

72. Zimmerman SB. Studies on the compaction of isolated nucleoids from Escherichia coli. J. Struct. Biol. 2004; 147:146-158. [PubMed: 15193643] 
73. Rivas G, Minton AP. Beyond the second virial coefficient: sedimentation equilibrium in highly non-ideal solutions. Methods. 2011; 54:167-174. [PubMed: 21112402]

74. Fernández C, Minton AP. Static light scattering from concentrated protein solutions. II. Experimental test of theory for protein mixtures and weakly self-associating proteins. Biophys. J. 2008; 96:1992-1998.

75. Gelman H, et al. ReAsH as a quantative probe of in-cell protein dynamics. Biochemistry. 2016; 55:1968-1976. [PubMed: 26959408]

76. Nagarajan S, et al. Modulation of functionally significant conformational equilibria in adenylate kinase by high concentrations of trimethylamine oxide attributed to volume exclusion. Biophys. J. 2011; 100:2991-2999. [PubMed: 21689533]

77. Rivas G, et al. Reconstitution of cytoskeletal protein assemblies for large scale membrane transformation. Curr. Opinion Chem. Biol. 2014; 22C:18-26.

78. Bakthisaran R, et al. Small heat shock proteins: role in cellular functions and pathology. Biochim. Biophys. Acta - Proteins and Proteomics. 2015; 1854:291-319.

79. Clegg JS. Properties and metabolism of the aqueous cytoplasm and its boundaries. Am. J. Physiol. 1984; 246:R133-R151. [PubMed: 6364846]

80. Aumiller WM Jr, Keating CD. Experimental models for dynamic compartmentalization of biomolecules in liquid organelles: reversible formation and partitioninng in aqueous biphasic systems. Adv. Colloid Interface Sci. 2016

81. Hartl FU, Hayer-Hartl M. Converging concepts of protein folding in vitro and in vivo. Nature Struct. and Mol. Biol. 2009; 16:574-581. [PubMed: 19491934]

82. Batra J, et al. Effect of macromolecular crowding on protein binding stability: modest stabilization and significant biological consequences. Biophys. J. 2009; 97:906-911. [PubMed: 19651049]

83. Kim YC, et al. Macromolecular crowding effects on protein-protein binding affinity and specificity. J. Chem. Phys. 2010; 133:205101. [PubMed: 21133453]

84. Phillip Y, et al. Common crowding agents have only a small effect on protein-protein interactions. Biophys. J. 2009; 97:875-885. [PubMed: 19651046]

85. Powers JD, et al. The predicted role of steric specificity in crowding-mediated effects on reversible biomolecular association. Physical Biology. 2015; 12:2-12.

86. Snoussi K, Halle B. Protein self-association induced by macromolecular crowding: a quantitative analysis by magnetic relaxation dispersion. Biophys. J. 2005; 88:2855-2866. [PubMed: 15665132]

87. Miklos AC, et al. Volume exclusion and soft interaction effects on protein stability under crowded conditions. Biochemistry. 2010; 49:6984-6991. [PubMed: 20672856]

88. Miklos AC, et al. Protein crowding tunes stability. J. Am. Chem. Soc. 2011; 133:7116-7120. [PubMed: 21506571]

89. Smith AE, et al. NMR studies of protein folding and binding in cells and cell-like environments. Current Opinion in Struct Biol. 2015; 30:7-16.

90. Ross PD, Minton AP. The effect of non-aggregating proteins upon the gelation of sickle cell hemoglobin: model calculations and data analysis. Biochem. Biophys. Res. Commun. 1979; 88:1308-1314. [PubMed: 38785]

91. Munishkina LA, et al. The effect of macromolecular crowding on protein aggregation and amyloid fibril formation. J. Mol. Recogn. 2004; 17:456-464.

92. Groen J, et al. Associative interactions in crowded solutions of biopolymers counteract depletion effects. J. Am. Chem. Soc. 2015; 137:13041-13048. [PubMed: 26383885]

93. McConkey EH. Molecular evolution, intracellular organization, and the quinary structure of proteins. Proc. Natl. Acad. Sci. USA. 1982; 79:3236-3240. [PubMed: 6954476]

94. Srere PA. Macromolecular interactions: tracing the roots. Trends in Biochemical Sciences. 2000; 25:150-153. [PubMed: 10694888]

95. Gnutt D, Ebbinghaus S. The macromolecular crowding effect - from in vitro into the cell. Biol. Chem. 2016; 397:37-44. [PubMed: 26351910]

96. Minton AP. The influence of macromolecular crowding and macromolecular confinement on biochemical reactions in physiological media. J. Biol. Chem. 2001; 276:10577-10580. [PubMed: 11279227] 
Text Box 1

\section{Thermodynamic basis of crowding effects on equilibria}

The physical origin of crowding effects upon macromolecular equilibria may be illustrated by two simple schemes indicating the free energy changes that accompany a two-state protein folding reaction (Figure IA) and the formation of a heterodimer (Figure IB) taking place in a dilute solution (upper rows) and a crowded solution (lower rows). Since free energy is conserved around a thermodynamic cycle, it follows that the difference between the free energies of folding in the crowded and dilute solutions (Fig. IA) is given by

$$
\Delta \Delta F_{U N}=\Delta F_{U N}-\Delta F_{U N}^{0}=\Delta F_{T, N}-\Delta F_{T, U}
$$

where $\Delta F_{T, X}$ denotes the change in the free energy (either Gibbs or Helmholtz) per mole of the system accompanying the transfer of dilute species $\mathrm{X}(=\mathrm{N}$ or $\mathrm{U})$ from a solution dilute in macromolecules to a solution containing arbitrary concentrations of one or more macromolecular solutes ("crowders"). Similarly, it follows that the difference between the free energies of heteroassociation in the crowded and dilute solutions (Fig. IB) is given by

$$
\Delta \Delta F_{A B}=\Delta F_{A B}-\Delta F_{A B}^{0}=\Delta F_{T, A B}-\left(\Delta F_{T, A}+\Delta F_{T, B}\right)
$$

where $\mathrm{X}=\mathrm{A}, \mathrm{B}$, or $\mathrm{AB}$. These are but two examples of the entirely general principle that for any reaction at equilibrium, if the sum of the free energy of transfer of all products is more negative than the sum of the free energy of transfer of all reactants, crowding biases the reaction toward products. Conversely, if the sum of the free energy of transfer of all reactants is more negative than the sum of the free energy of transfer of all products, crowding biases the reaction toward reactants ${ }^{12}$. This relationship is independent of the absolute values of the free energies of transfer, which may be either positive or negative.

We define the apparent equilibrium constants $K_{U N}=[N] /[U]$ and $K_{A B}=[A B] /[A][B]$. The effect of crowding by a volume fraction $\phi$ of crowder upon these equilibrium constants may be quantified as $\ln \Gamma_{U N}(\phi)=\ln \left(K_{U N}(\phi) / K_{U N}^{0}\right)$ and $\ln \Gamma_{A B}(\phi)=\ln \left(K_{A B}(\phi) / K_{A B}^{0}\right)$, where the superscripted equilibrium constants denote the values of the respective constant in dilute solution. For chemical reactions at constant pressure, where $\Delta F$ is equal to the Gibbs free energy $\Delta G$, the magnitude of the crowding effect is a function of the crowding-induced changes in Gibbs free energy $(\Delta \Delta G)$, enthalpy $(\Delta \Delta H)$, and entropy $(\Delta \Delta S)$ of each reaction:

$$
\ln \Gamma_{U N}=\frac{\Delta \Delta G_{U N}}{R}\left(\frac{1}{T}\right)=-\frac{\Delta \Delta H_{U N}}{R}\left(\frac{1}{T}\right)+\frac{\Delta \Delta S_{U N}}{R}
$$


and

$$
\ln \Gamma_{A B}=\frac{\Delta \Delta G_{A B}}{R}\left(\frac{1}{T}\right)=-\frac{\Delta \Delta H_{A B}}{R}\left(\frac{1}{T}\right)+\frac{\Delta \Delta S_{A B}}{R}
$$

It is evident from equations [2ab] that enthalpic contributions to the total crowding effect will be manifested as a temperature dependence of the magnitude of the crowding effect, and conversely, the lack of a measurable temperature dependence of the magnitude of the crowding effect will indicate the lack of a significant enthalpic contribution. 
A
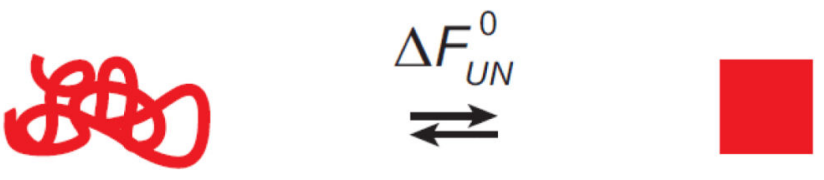

$\uparrow \Delta F_{T, U}$
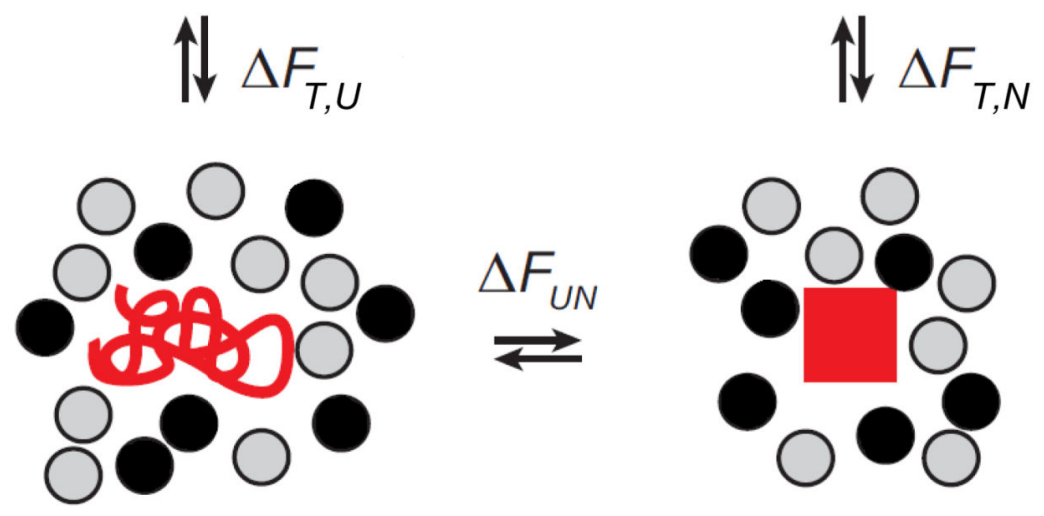

B.

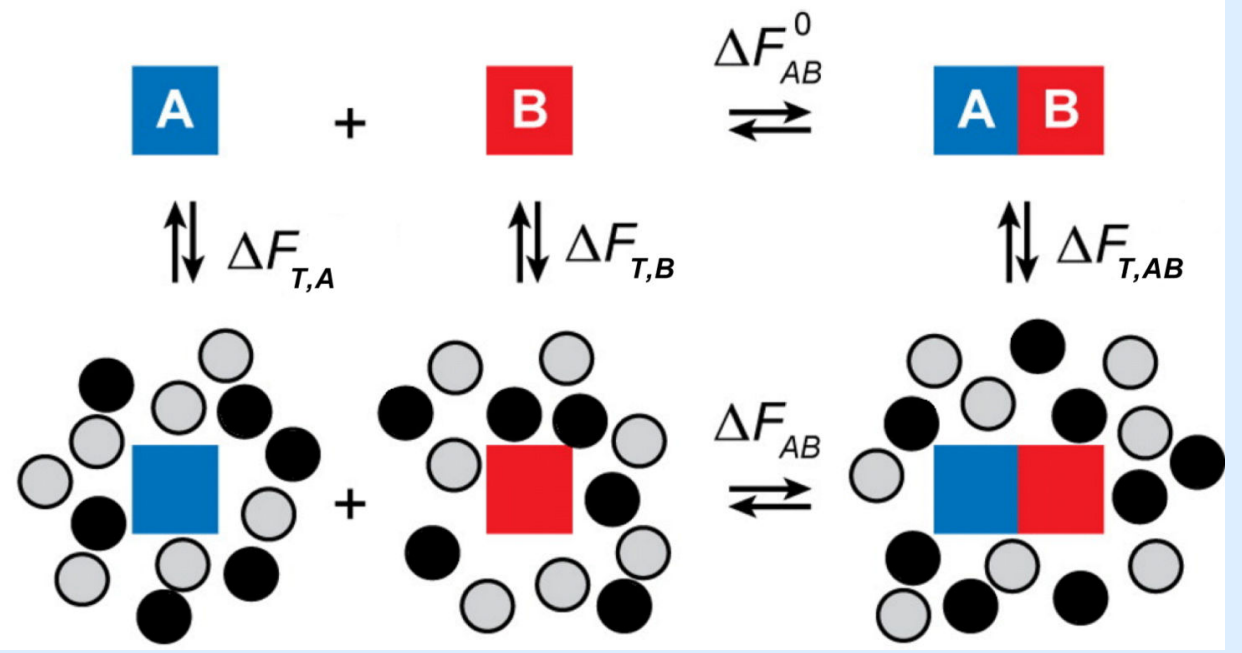

Figure I. Thermodynamic cycles illustrating the origin of the difference between the free energy of folding (Panel A) and binary heteroassociation (Panel B) in a dilute solution (upper row) and a crowded solution (lower row). Figures adapted from ${ }^{14}$.

Trends Biochem Sci. Author manuscript; available in PMC 2018 February 08. 
Text Box 2

\section{Recommendations for design of an interpretable crowding experiment}

1. Crowding agent should be significantly larger than solvent, so that equivalent hard particle models can be validly used to estimate magnitude of excluded volume effects.

2. The behavior of the crowding agent at high concentration should be characterized to ensure the absence of crowder self-association or aggregation at high concentration.

3. Co-sedimentation assays should be performed to verify the absence of crowder-tracer association.

4. The reaction equilibrium constant in the absence of added crowder should be poised sufficiently close to unity, so that if crowding effects supply a significant increment of free energy, the change in species abundance will be experimentally measurable. Poising can be accomplished by adjusting the $\mathrm{pH}$ or ionic strength, or by adding destabilizing reagents such as urea or stabilizing reagents such as TMAO.

5. The effect of the crowder on the reaction equilibrium should be measured over a range of temperatures in order to ascertain the relative effect of the crowder on the enthalpy change and entropy change of the reaction.

6. Experiments aimed at characterizing changes in conformational equilibria should be conducted at multiple reactant concentrations to detect possible crowder-induced self-association of the reactant that, if present, would complicate interpretation of the results.

7. If multiple crowding agents are utilized, the mixture of agents should be characterized as a function of composition and total crowder concentrations in order to verify the absence of self- and/or hetero-association. (Crowder selfor heteroassociation reduces the volume exclusion effect of the crowder per unit w/v concentration ${ }^{13}$.)

8. Experiments should be conducted with more than one concentration of crowding agent, to ascertain whether or not the effect observed is qualitatively or semiquantitatively consistent with the predictions of excluded volume theory.

9. Experiments should be conducted with more than one crowding agent to ascertain the the extent to which results agree with the predictions of excluded volume theory. Globular proteins are to be preferred over random coil polymers as crowding agents, as excluded volume theory of polymer-polymer and polymer-protein interactions is primitive at best. 


\section{Outstanding Questions Box}

How and why do crowding effects vary from point to point within a cell?

Is crowding more important in some intracellular microenvironments/organelles/ compartments than others?

How strongly do non-crowding interactions between soluble macromolecules and structural elements of the cell affect the transport properties and reactivity of the soluble species in an intact cell?

Is it possible to design a true "crowding sensor" that can distinguish between the effects of crowding in an unperturbed microenvironment and tracer-induced interactions? 


\section{Trends}

- $\quad$ Recent publications emphasize contributions of both steric repulsion and longer-ranged attractive and repulsive intermolecular interactions to the total effects of crowding upon the conformational and association equilibria and kinetics of proteins and nucleic acids.

- Quantitative studies of the colligative properties of protein/protein and protein/polymer mixtures have provided new information about the self- and hetero-interactions between macromolecules that govern macromolecular behavior in concentrated and/or crowded solutions.

- It is becoming increasingly recognized that the results of tracer experiments conducted on labeled proteins in intact cells cannot be interpreted unambiguously until augmented by comparable experiments conducted using the same and other tracers within homogeneous and well-characterized cellular microcompartments.

- We suggest that colleagues abstain from untested claims of general validity of results obtained with a particular system under a single set of conditions. 

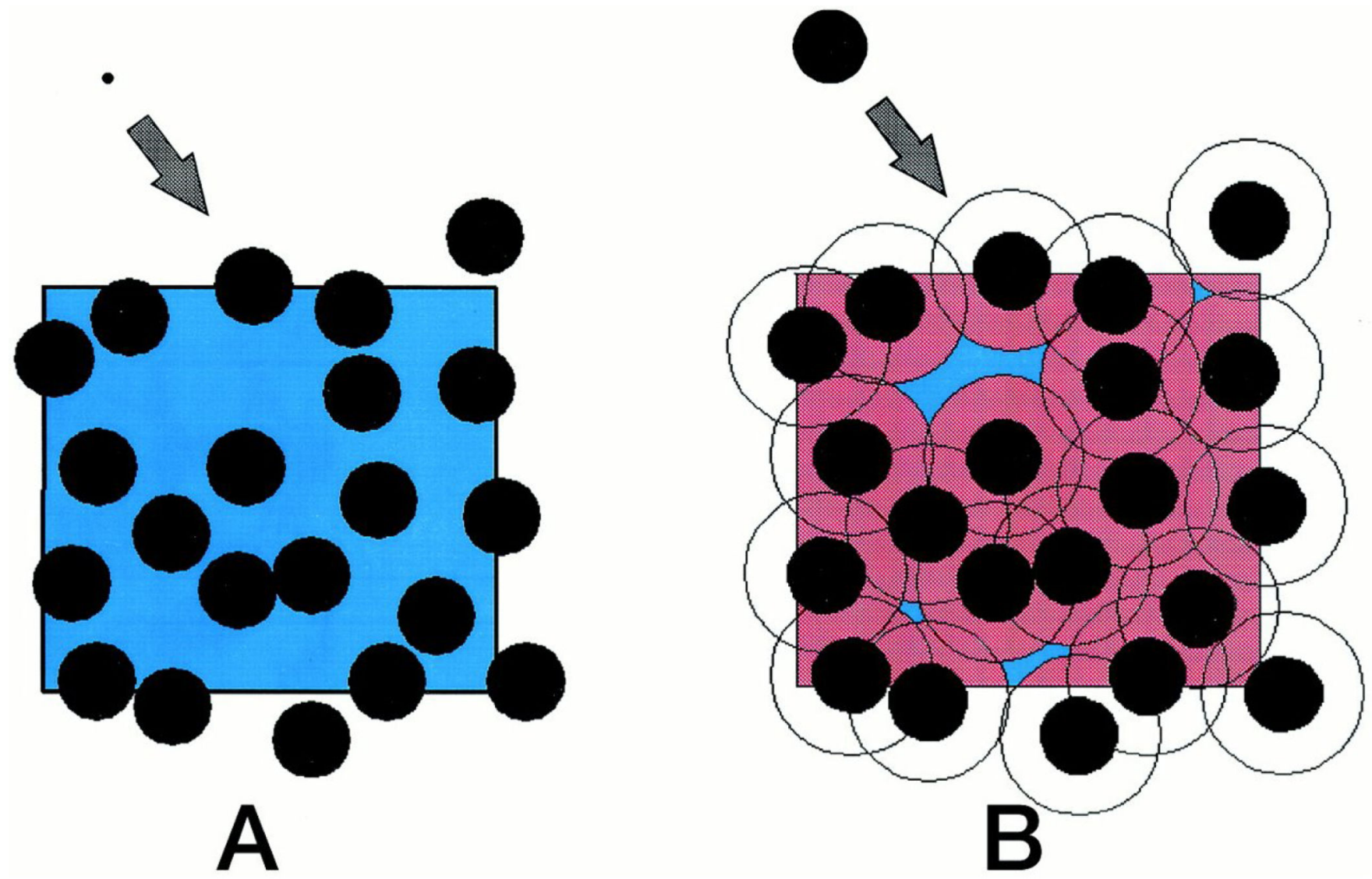

Figure 1.

Schematic depiction of volume instantaneously excluded [pink] and available (= total excluded) [blue] to the center of mass of a newly added molecule that is small (panel A) and comparable in size (panel B) relative to the concentrated macromolecule. Excluded volume contributes $R T \ln \left(v_{\text {total }} / V_{\text {available }}\right)$ to the total free energy of interaction between the added molecule and the concentrated molecules. Figure reproduced with permission from ${ }^{96}$. 


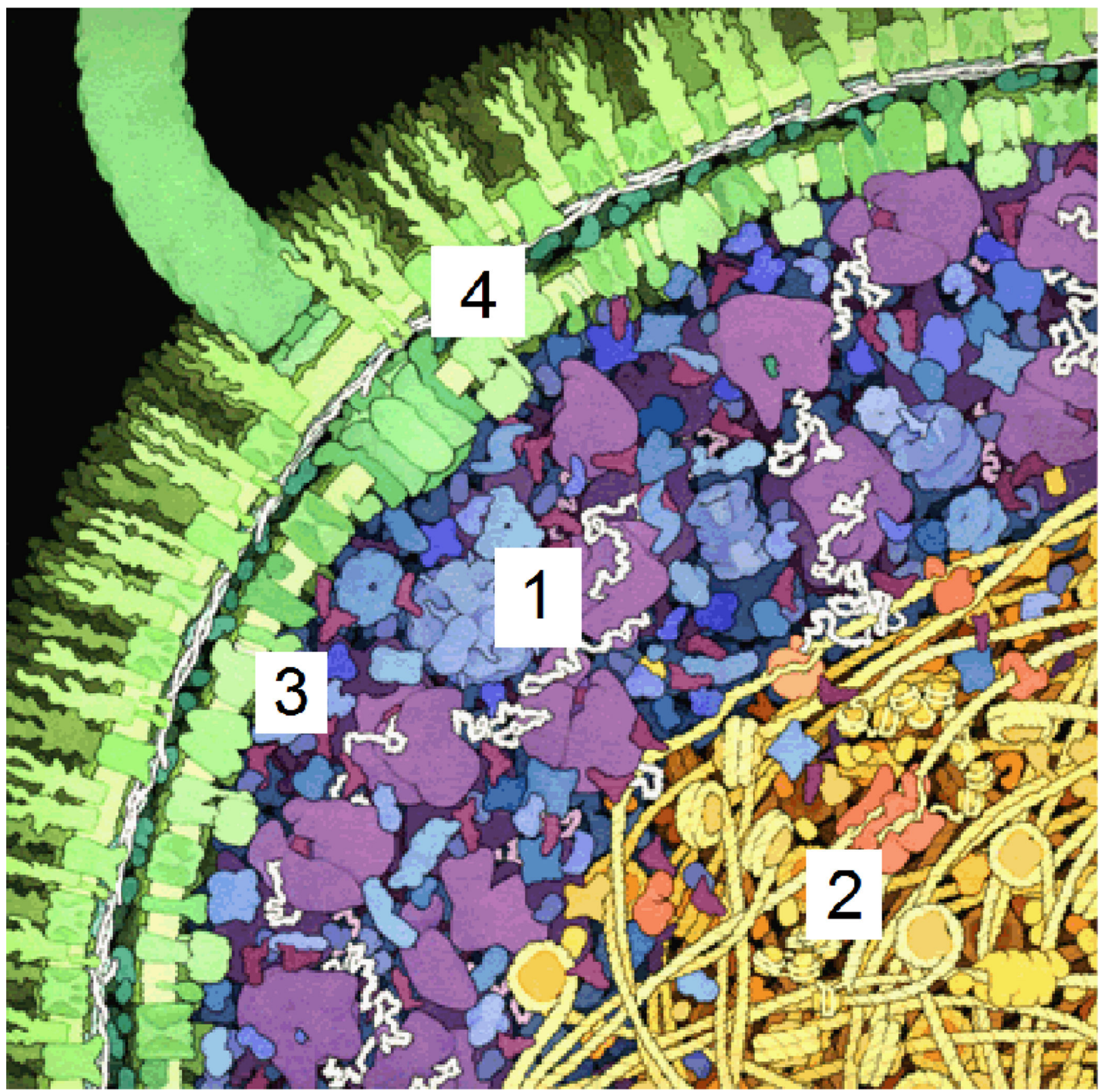

Figure 2.

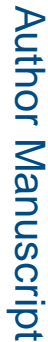

Cartoon of the interior of an E. coli cell. Four distinct microcompartments are indicated: (1) The cytoplasm exterior to the nucleoid, consisting of primarily soluble proteins, ribonucleic acids, and macromolecular assemblies such as ribosomes and proteasomes; (2) The interior of the nucleoid, with an extremely high local concentration of DNA and DNA-binding and DNA-condensing proteins; (3) The region immediately adjacent to the inner plasma membrane, containing a high local concentration of membrane lipids and intrinsic membrane proteins, and (4) the periplasm, containing high local concentrations of 
membrane proteins and interstitial proteoglycans. Figure adapted from Goodsell (http:// mgl.scripps.edu/people/goodsell/illustration/public). 
A

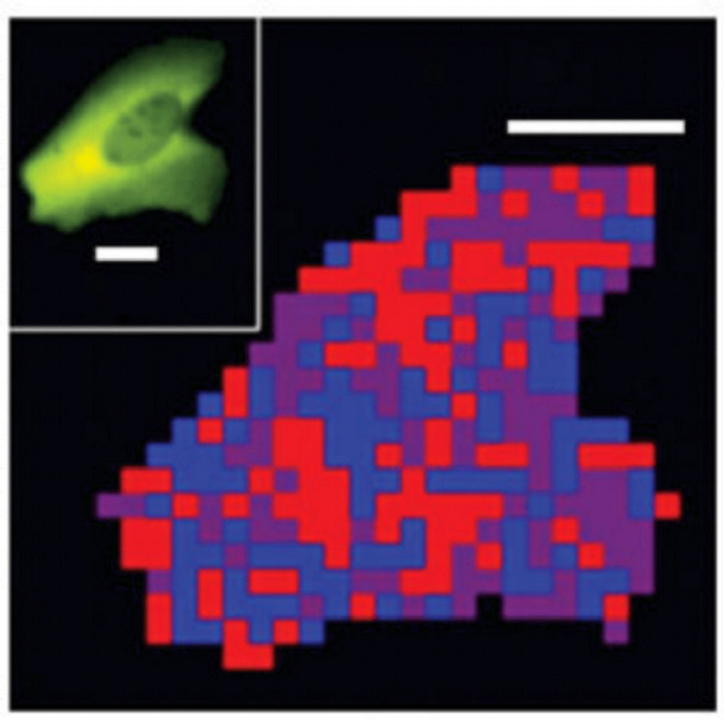

B

\section{Melting temperature}

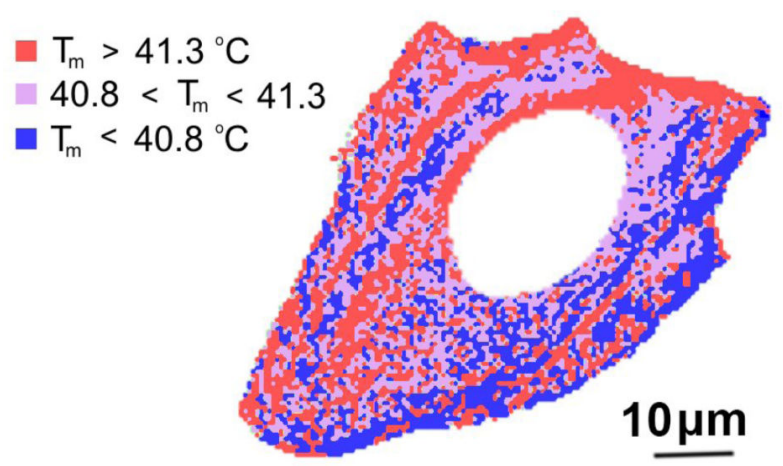

Figure 3.

Positional variability in folding kinetics and equilibria. A - Positional variation of folding relaxation time $\tau$. blue: $\tau<2.7 \mathrm{~s}$, violet: $2.7 \mathrm{~s}<\tau<3.2 \mathrm{~s}$, red: $\tau>3.2 \mathrm{~s}$. Scale bar: $10 \mu \mathrm{m}$. Figure reproduced from ${ }^{55}$ with permission. B - Positional variation of melting temperature. Scale bar: $10 \mu \mathrm{m}$. Figure adapted from ${ }^{65}$. 